\section{BÜCHER KURZ VORGESTELLT}

\section{BERND MARTENS: Die gesellschaftliche Resonanz auf das Abfallproblem. Wiesba- den: DUV Deutscher UniversitätsVerlag, 1999. 251 S. DM 58,--. ISBN 3-8244-4338-4}

Die Arbeit ist in einen theoretischen und einen empirischen Hauptteil gegliedert. Zunächst wird in einer historischen Übersicht die Entwicklung der Abfallproblematik dargestellt. Es werden drei Phasen unterschieden, wobei der noch andauernde dritte Zeitabschnitt durch Tendenzen der Verwissenschaftlichung, Verrechtlichung und Ökonomisierung des Entsorgungssystems gekennzeichnet ist. Im theoretischen Kapitel wird eine primär wissenssoziologische Sichtweise zugrunde gelegt, bei der Fragen der Definitionsmacht über thematische Rahmungen von Situationen und kollektive Zumutbarkeiten von Risiken eine Rolle spielen.

Die empirischen Teile beziehen sich auf vier verschiedene Datengrundlagen: Datenreihen, die im Rahmen der amtlichen Statistik erhoben wurden; Leitfadeninterviews mit Mitarbeitern aus Abfallwirtschaftsämtern; Wahrnehmungen und Präferenzen von Akteuren, die durch eine Befragung erhoben wurden, und Themen des öffentlichen Diskurses über das Abfallproblem, wie er sich in Inhalten von Zeitungsartikeln niederschlägt. Einige Ergebnisse der empirischen Analysen sind:

- Es ist zu einer teilweisen Entkopplung von Wirtschaftswachstum und Abfallproduktion gekommen; ob dies aber angesichts eingeschränkter Handlungsmöglichkeiten individueller Abfallproduzenten wirklich eine "Trendwende" darstellt, ist strittig.

- Der Dissens zwischen unterschiedlichen Akteuren bei abfallwirtschaftlichen Fragen ist abhängig von der kognitiven Sicherheit, mit der Auffassungen vertreten werden, und von dem Misstrauen anderen Akteuren gegenüber.

- Die Beratung der Öffentlichkeit durch Experten über die Bürden eines "richtigen" Abfallverhaltens ist $\mathrm{zu}$ einer wichtigen thematischen Rahmung in der Presse geworden.
Abschließend werden mögliche zukünftige Entwicklungen der Abfallproblematik in die augenblickliche Diskussion über Nachhaltigkeit eingeordnet.

(Verlagsankündigung)

Einen zusammenfassenden Überblick zu diesem Buch stellt Martens unter dem Titel "Gesellschaftliche Resonanz auf das Abfallproblem: Wahrnehmungen und Thematisierungsprozesse in einer historischen Perspektive" im Schwerpunktthema dieser Ausgabe der TA-Datenbank-Nachrichten vor.

RENATE MARTINSEN, GEORG SIMONIS (Hrsg.): Demokratie und Technik - (k)eine Wahlverwandtschaft? Opladen: Leske + Budrich, 2000. 436 S. DM 80,--. ISBN 3-8100-2473-2

Die Problemstellung "Demokratie und Technik" ist nicht neu. Doch ist es für die Politikwissenschaft unvermeidlich, sich mit ihr von Zeit zu Zeit erneut $\mathrm{zu}$ befassen. Nicht nur verändert sich die Realität: die Demokratie, die Technik und ihre Wechselbeziehungen, sondern auch der wissenschaftliche Blick: die disziplinäre Konstruktion, analytische Dekonstruktion und normative Orientierung. So lässt sich bilanzieren, dass wir nach den Debatten über den "Technischen Staat" (60er Jahre), die Möglichkeiten und Grenzen staatlicher Technologiepolitik (70er Jahre), die Chancen und Risiken der Informationsgesellschaft (80er Jahre), die Steuerungsfähigkeit und mögliche Steuerungsformen des Staates im Mehrebenensystem (Ende der 80er/Anfang der 90er Jahre) seit Mitte der 90er Jahre in eine neue Phase der Diskussion des Verhältnisses von Politik und Technik eingetreten sind, in der demokratietheoretische Fragestellungen wieder einen höheren Stellenwert bekommen haben. Der vorliegende Band gibt einen Einblick in die gegenwärtig geführte Debatte und ist seismographisch für zukünftige Problemstellungen.

Nach einem Vorwort von R. Martinsen und G. Simonis ist der Band wie folgt gegliedert: 
- BügerInnen, Technik und Demokratie (Th. Saretzki, R. Martinsen)

- Demokratieverträgliche Technikentwicklung? (O. Winkel, M. Mai, St. Albrecht)

- Demokratiedefizite auf der europäischen Ebene (M. Behrens/G. Simonis/R. Droz)

- Resonanzfähigkeit der Politik als Voraussetzung innovativer Technikregulierung (V. M. Brennecke, R. Herbold/W. Krohn/ M. Timmermeister, M. Gsänger)

- Instrumente reflexiver Techniksteuerung (H. J. Kleinsteuber, St. Kuhlmann)

- Das demokratische Potential interaktiver Medien (P. Mambrey, V. Leib, R. Wirth, H. Geser)

(Verlagsankündigung)

》

MICHAEL ORNETZEDER: Die Solaranlage Soziale Genese einer zukunftsfähigen Technik. Frankfurt/M. (u. a.): Peter Lang Verlag, 2000. (Sozialwissenschaftliche interdisziplinäre Studien, Bd. 4) 177 S. DM 65,--. ISBN 3-631-35244-1

In diesem Buch wird die Entstehung der thermischen Solaranlage aus soziologischer Perspektive rekonstruiert. Es handelt sich überraschenderweise um eine alte Technik, entwickelt im ausgehenden 19. Jahrhundert in den USA. Nach anfänglichen Verbreitungserfolgen wird sie jedoch durch die Konkurrenz von Gas- und Elektrizitätsgesellschaften wieder vom Markt verdrängt und in Europa erst nach dem sogenannten Ölschock im Jahr 1973 als umweltfreundliche Alternative aufgegriffen. Während die Entstehung der Solaranlage durch eine Reihe gesellschaftlicher Bedingungen begünstigt wurde, kann deren weitere Genese in erster Linie anhand der Strategien und Möglichkeiten relevanter sozialer Akteure beschrieben werden. Interessant ist, dass die - aus heutiger Sicht wichtigen - ökologischen Vorzüge der Solartechnik sich damals eher negativ auf deren Weiterentwicklung auswirkten.

(Verlagsankündigung)

》
DIRK SCHLESINGER: Die Liberalisierung des Marktes für Elektrizität in der Bundesrepublik Deutschland und ihre ökologischen Folgen - dargestellt anhand einer raumzeitlichen Modellrechnung unter Berücksichtigung volkswirtschaftlicher und strukturpolitischer Rahmenbedingungen. Mannheimer Geographische Arbeiten, Heft 50, 1999. DM 35,--. ISBN 3-923750-77-3

Die von der Europäischen Union seit 1990 angeregte und Ende des Jahres 1997 in der Bundesrepublik Deutschland auf nationaler Ebene zur Umsetzung beschlossene Richtlinie zur Deregulierung des Strommarktes bewirkt eine tiefgreifende Änderung der Wettbewerbsregeln in der Elektrizitätswirtschaft. Die ökologischen Folgen der Deregulierung werden jedoch kontrovers diskutiert, die diesbezüglichen Aussagen sind oft widersprüchlich.

So wird argumentiert, dass der durch die Deregulierung ausgelöste Preis- und Kostendruck die Möglichkeiten der Energieversorger $\mathrm{zu}$ umweltschonenden Investitionen einschränkt. Allerdings führt derselbe Kostendruck zu verstärkter Substitution von Kohlekraftwerken durch kombinierte Gas- und Dampfkraftwerke, die sich durch sehr hohe Wirkungsgrade und geringe spezifische Emissionen auszeichnen. Somit erhöht sich die Abhängigkeit vom importierten Primärenergieträger Gas, welcher mit einer größeren Preisvolatilität als Kohle behaftet ist. Weiterhin sind die nach der Deregulierung niedrigeren Strompreise zwar positiv für die Wettbewerbsfähigkeit des Industriestandortes Deutschland, doch fördern sie gleichzeitig den Einsatz von Strom zur Gewinnung von Prozess- und Heizwärme. Aufgrund des geringeren Konversionsgrades von Primärund Nutzenergie wird eine größere Menge an Emissionen frei als beim direkten Einsatz fossiler Energieträger zu Heizzwecken.

Kernstiuck der vorliegenden Arbeit ist die Quantifizierung der ökologischen Folgen der Deregulierung des Elektrizitätsmarktes, d.h. die Prognose der Entwicklung des $\mathrm{CO}_{2^{-}}, \mathrm{SO}_{2^{-}}$, $\mathrm{NO}_{\mathrm{x}}$ - und NMVOC-Emissionen des deutschen Kraftwerksparks bis zum Jahr 2025. Dazu wird ein für diese Arbeit entwickeltes numerisches Simulationsmodell verwendet. Ebenfalls diskutiert werden ökonomische Folgen wie die Entwicklung des Primärenergiemix und des Kostengefüges der Stromerzeugung sowie indust- 
riegeographische Folgen für ausgewählte Regionen Deutschlands.

Nach der ausführlichen Darlegung der Ausgangsbedingungen für die Deregulierung des deutschen Strommarktes wird das in der Studie verwendete Simulationsmodell vorgestellt, wobei - im Unterschied zu bisherigen Simulationsmodellen, welche von einer Organisation des Elektrizitätsmarktes als Pool-Modell ausgehen - hier der Stromhandel zwischen unabhängigen Energieversorgern unter SingleBuyer mit frei verhandeltem Netzzugang Dritter (Negotiated Third Party Access, NTPA) abgebildet wird. Daran anschließend werden 64 Szenarien definiert, die - wegen deren teilweiser Redundanz - letztlich zu 40 verschiedenen zu berechnenden Szenarien führen. sind:

Die wichtigsten Ergebnisse der Analyse

Ökologisch wirkt sich die Deregulierung positiv aus, da die auf eine produzierte $\mathrm{kWh}$ bezogenen Emissionen des deutschen Kraftwerksparks langfristig abnehmen. Um trotz der für die Zukunft prognostizierten Zunahme der Stromnachfrage eine absolute Reduzierung der emittierten Schadstoffmengen zu erzielen, sind jedoch Eingriffe in Form einer emissionsmengenabhängigen Steuer notwendig. Ein gradueller Rückzug aus der Stromerzeugung mit Kernkraft führt zu höheren Emissionen, welche sich bei niedrigen Gaspreisen durch eine $\mathrm{CO}_{2}$-Steuer jedoch größtenteils ausgleichen ließen. Andere Faktoren wie verstärkte Stromimporte, unterschiedliche Péage-Höhen und die Anzahl von Elektrizitätsversorgungsunternehmen im Markt zeigen im Vergleich zu den Einflussfaktoren Gaspreis, Steuerregime und Kernkraftausstieg nur geringe Wirkung auf die deutsche Emissionsbilanz.

Ökonomisch betrachtet wird die in Deutschland eingeführte Form der Deregulierung sowohl dem Ziel der Verringerung der Erzeugungskosten als auch dem Ziel der Homogenisierung der Erzeugungskosten gerecht. Die gehandelten Strommengen liegen dabei in einer Größenordnung, welche eine grundlegende Neustrukturierung des deutschen Höchstspannungs-Transportnetzes unnötig erscheinen lässt. Eine mit Einführung einer $\mathrm{CO}_{2}$-Steuer vor allem bei niedrigem Gaspreis stattfindende Substitution von Braun- und Steinkohle durch Gas bewirkt eine starke Steigerung der Gas- nachfrage der deutschen Elektrizitätswirtschaft. Aussagen darüber, in welchem Maße sich ein solcher Anstieg importierter Erdgasmengen auf das Primärenergiepreisgefüge auswirkt und eine Verdrängung von Kohle als einzigem in großen Mengen vorhandenen heimischen Primärenergieträger politisch toleriert wird, sind nicht Bestandteil dieser Arbeit.

Regional betrachtet gilt allgemein, dass sich der Strompreis und die Lage der Unternehmen an großen Wasserwegen für den Transport oder in Nähe der Kohlevorkommen entkoppeln und sich damit Unterschiede für energieintensive Industrien bezüglich des wichtigen Standortfaktors Strompreis egalisieren. Um gezielte Aussagen über die Folgen der Deregulierung auf einzelne Regionen machen zu können, ist eine genaue Analyse des energiewirtschaftlichen und industriellen Umfeldes der jeweiligen Region notwendig. So hat z.B. eine $\mathrm{CO}_{2}$-Steuer je nach Primärenergieeinsatz eines Erzeugers spezifische Auswirkungen auf dessen Kraftwerkspark und Wettbewerbsumfeld sowie auf die in die Wertschöpfungskette der Energieversorgung eingebundenen Industrien.

\section{Bestellungen bitte richten an:}

MANNHEIMER GEOGRAPHISCHE ARBEITEN Geographisches Institut der Universität Mannheim D-68131 Mannheim

Fax: + 49 (0) $621 / 181-1955$

E-Mail: gimga@rumms.uni-mannheim.de

\section{ERIKA DÖRNER: Bibliographische Umwelt- aspekte der Gentechnik. Bearb.: Erika Dör- ner, Astrid Schubert. Hrsg. vom Umwelt- bundesamt. Berlin: Erich Schmidt Verlag, 1999. 608 S. DM 98,--. ISBN 3-503-05835-4}

Seit der Verabschiedung des Gentechnikgesetzes im Jahr 1990 hat sich die wissenschaftliche Forschung auf dem Gebiet der Gentechnik rasant entwickelt. Die Vielzahl der Anwendungsbereiche der Gentechnik umfasst neben Medizin, Pharmazie, Umweltschutz und Lebensmittel auch die sogenannte "Grüne Gentechnik", welche sich mit Züchtung und Anbau gentechnisch veränderter Nutzpflanzen beschäftigt und 
in dieser Bibliographie einen breiten Raum einnimmt.

Im Gegensatz zu anderen Wissenschaftszweigen ist die Gentechnik ein relativ junges Gebiet, welches neben der Informations- und Kommunikationstechnik unsere Gesellschaft zukünftig wesentlich prägen wird.

Die Diskussion über die Anwendung der Gentechnik und ihre Folgen wird nach wie vor kontrovers geführt. Entsprechend hoch ist demzufolge das Bedürfnis der Menschen nach umfassender, aktueller Information. Die vorliegende Bibliographie versucht, diesem Anspruch gerecht zu werden. Sie umfasst eine breite $\mathrm{Pa}$ lette relevanter Publikationen, die neben Grundlagenforschung und Übersichtswerken schwerpunktmäßig vor allem Sicherheits- und Begleitforschung, ökologische Themen sowie ethische und gesellschaftspolitische Fragen behandeln.

Die Gentechnik-Bibliographie wendet sich an einen relativ breiten Nutzerkreis in Forschungseinrichtungen, Verwaltung, Politik, Wirtschaft, Umweltverbänden und nicht zuletzt an die Verbraucher.

Insgesamt umfasst die Bibliographie rund 1.800 Fachveröffentlichungen aus der ULIDAT (Umweltliteraturdatenbank), rund 250 Forschungs- und Entwicklungsvorhaben aus der UFORDAT (Umweltforschungsdatenbank) sowie 68 Einträge aus der URDB (Umweltrechtsdatenbank).

Berücksichtigt wurden Publikationen ab 1995, Forschungsvorhaben aus der UFORDAT schon ab 1994. Neben überwiegend deutschsprachigen Veröffentlichungen wurden auch eine Vielzahl Publikationen aus dem europäischen Raum sowie den USA ausgewählt. Etwa $25 \%$ der Veröffentlichungen sind englischsprachig.

Die genannten Datenbanken des UBA sind auch online zugänglich. Informationen hierzu bei Umweltbundesamt, Postfach 3300 22, D-14191 Berlin, Tel.: + 49 (0) 30 / 8903-0, Fax: +49 (0) 30 / 89032102.

Internet: http://www.umweltbundesamt.de

(aus dem Vorwort) 